\title{
Annual Congress of the Chinese Blood Purification Center Administration Committee
}

August 20-22, 2015, Tianjin, China

\section{Selected Abstracts}

Guest Editor

Tao Wei, Beijing 


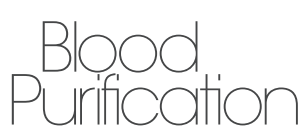

1

\section{The Creation of a Canine Arteriovenous Fistula Model and Preliminary Experimental Study}

\author{
Wang Bo, Fang wei, Li-hua wang, Zhi Lu, Lan Jia, \\ Ai-li Jiang* \\ Department of Kidney Disease and Blood Purification \\ The Second Hospital of Tianjin Medical University, \\ Tianjin, China \\ *Corresponding author: Ai-li Jiang \\ E-Mail: ailij@163.com
}

Objective: This study anastomosed femoral artery and vein of domestic dogs to creat arteriovenous fistula animal model and validated this is an ideal model in which to test out novel interventions for the prevention and treatment of clinical hemodialysis vascular access dysfunction. At the same time we carried out preliminary experiments, we measured and recorded blood flow volume, vascular diameter, vascular neointima thickness, serum endothelin-1 and nitric oxide concentrations at different time.

Methods: 12 dogs were created arteriovenous fistula though anastomosed femoral artery and vein. Fasting blood samples were taken on the day of surgery, postoperative 3 days, one week, two weeks, four weeks, the ELISA method were used to test the serum endothelin-1 and nitric oxide concentrations. At the same time using color Doppler imagining clearly show anastomotic arterial and venous side of its main vessels, measuring its diameter, measuring anastomotic diameter and calculating peak blood flow. 6 dogs were sacrificed 7 days after the operation, the others were sacrificed 28 days after the operation. The femoral vein were harvested after the canine were sacrificed and the change of the neointima were observed by microscope. Application of SPSS 17.0 statistical package was used for statistical analysis of all data, $\mathrm{P}<0.05$ was considered statistically significant.

Results: (1) All the operation were succeed, peak blood flow reached $364.77 \pm 31.00 \mathrm{ml} / \mathrm{min} 7$ days after the operation and all the arteriovenous fistula matured well. (2) The femoral artery and femoral vein were marked obvious enlarged 3 days after the operation, and tended to progress gradually with time. (4) NO increased obviously 3 days after the operation, and were significantly higher than other groups $(\mathrm{P}<0.05)$. Intercomparison in other groups no significant difference were observed $(\mathrm{P}>0.05)$. The concentration of ET-1 achieved peak point 14 days after the operation. Compared with the day of surgery, ET-1 increased significantly at 7, 14, 21 days after the surgery $(\mathrm{P}<0.05)$. Intercomparison in other groups no significant difference were observed $(\mathrm{P}>0.05)$. (5) Neointima hyperplasia were observed 7 days after the operation and its' thickness were $25.32 \pm 3.96 \mathrm{um}$. Neointima hyperplasia tended to progress gradually with time and neointima thickness achieved $195.77 \pm 27.51 \mathrm{um}$ at day 28 after the operation.
Conclusions: (1) We have developed and validated a canine model of venous neointimal hyperplasia that is very similar to the human lesion. We believe that this is an ideal model in which to test out novel interventions for the prevention and treatment of clinical hemodialysis vascular access dysfunction. (2) In the early stage after the surgery, the concentration of NO increased obviously, it benefited vascular remodeling and benefited arteriovenous fistula mature.7 days after the surgery neointimal hyperplasia began to happen, the concentration of ET-1 also increased, maybe it has a close relationship with the neointimal hyperplasia. (3) After the surgery the concentration of NO and ET-1 changed and this may provided theoretical foundation for future research.

Key Words: Arteriovenous fistula; Neointimal hyperplasia; Nitric oxide; Endothelin-1; Animal model.

\section{2}

\section{Role of cPKC $\beta$ II/Akt Signaling Pathway on Low Plasma $\mathrm{H}_{2} \mathrm{~S}$ Predicted Cardiovascular Death in Chronic Hemodialysis Patients}

\author{
Han Li, Su-juan Feng, Shi-xiang Wang* \\ Department of Blood Purification, Beijing Chao-Yang \\ Hospital, Capital Medical University, Nephrology Faculty، \\ Capital Medical University, Beijing, China \\ * Correspondence author: Shi-xiang Wang \\ E-Mail:wxy1988@263.net
}

This work was supported by National Natural Science Foundation of China (81200543), Beijing Natural Science Foundation (7142057) and Beijing Municipal Health Bureau High-level Medical Professionals Promotion Project (2013-3-016).

Objective: Considering the high motality induced by cardiovascular diseases in chronic kidney disease (CKD) patients and the cardiovascular protective effect of $\mathrm{H}_{2} \mathrm{~S}$, the aim of this study was to evaluate the relationship between plasma $\mathrm{H}_{2} \mathrm{~S}$ and cardiovascular risk markers, including pulse pressure (PP), left ventricular mass index (LVMI), intima-media thickness (IMT) and carotid-femoral PWV $\left(\mathrm{PWV}_{\mathrm{CF}}\right)$, and mortality in chronic hemodialysis (CHD) patients. And further investigate the underlying cardiovascular protection mechanism of $\mathrm{H}_{2} \mathrm{~S}$ in $\mathrm{CHD}$ patients with uremic accelerated atherosclerosis (UAAS).

Methods: 113 CHD patients (62 men, 51 women) who had no residual renal function and had undergone regular dialysis treatment for at least 3 months were enrolled into this study. Plasma $\mathrm{H}_{2} \mathrm{~S}$ was measured through zinc acetate reaction. cPKCßII membrane translocation and phosphorylation of Akt were detected by Western blot. The ICAM-1 and VCAM-1 levels were detected by ELISA.

Results: Lower plasma $\mathrm{H}_{2} \mathrm{~S}$ level in CHD patients was predictor of an increased PP, LVMI and IMT. Patients with lower $\mathrm{H}_{2} \mathrm{~S}$ had a

\section{KARGER \\ (C) 2016 S. Karger AG, Basel \\ 0253-5068/16/0414-0294\$39.50/0}


lower survival at the end of the study. $\mathrm{H}_{2} \mathrm{~S}$ was an independent predictor of all-cause and cardiovascular mortality when adjusted for other risk factors. CHD Patients with lower $\mathrm{H}_{2} \mathrm{~S}$ showed an increase of cPKCßII activation, but phosphorylation of Akt decreased. The level of VCAM-1 and ICAM-1 increased significantly.

Conclusions: These results obtained confirm that lower plasma $\mathrm{H}_{2} \mathrm{~S}$ concentration in CHD patients is negative correlated with many cardiovascular risk factors such as PP, LVMI and IMT and high mortality, which may mediated by the cPKCBII/Akt signaling pathway and further its potential downstream substrates VCAM-1 and ICAM-1 is upregulation.

Key Words: Hemodialysis; Uremic accelerated artherosclerosis; Hydrogen sulfide; Mortality; Conventional protein kinase C $\beta \mathrm{II}$; Akt.

\section{3 \\ Risk Factors of Mortality in Acute Kidney Injury Patients Receiving Continuous Renal Replacement Therapy after Cardiac Surgery}

\author{
Zuan-hong Jiang, Zi-jin Chen, Ping Li, Xiao-bo Ma, \\ Hai-jin Yu, Xiao-nong Chen* \\ Department of Nephrology, Ruijin Hospital, Shanghai \\ Jiaotong University School of Medicine, Shanghai, China \\ ${ }^{*}$ Corresponding author: Xiao-nong Chen \\ E-Mail: xiaonong@medmail.com.cn
}

Objective: To explore the risk factors of mortality in patients suffering from acute kidney injury (AKI) after cardiac surgery and undergoing continuous renal replacement therapy (CRRT).

Methods: A total of 78 patients who suffered from AKI after cardiac surgery and later were treated with continuous veno-venous hemodiafiltration (CVVHDF) in Ruijin Hospital of Shanghai Jiao Tong University from September 2007 to January 2013 were enrolled in this study. Among the 78 patients, 55 were male and 23 were female; 44 were dead and 34 were survival while discharging. Demographic data, biochemical data before and after the surgery were collected and analyzed. Left ventricular ejection fraction (LVEF) of each patient was evaluated before the surgery. Logistic regression analysis was applied to analyze independent risk factors of mortality in AKI patients after cardiac surgery.

Results: There were no significant differences in terms of age, gender, hypertension, diabetes, chronic kidney disease, cerebrovascular disease, NHYA heart function, cardiopulmonary bypass, transfusion during the surgery, estimated glomerular filtration rate (eGFR), or LVEF between survival group and death group $(\mathrm{P}>0.05)$. The interval time between cardiac surgery and CVVHDF of the survival group was significantly shorter than that of death group $(\mathrm{P}<0.05)$. Preoperative hemoglobin, albumin and calcium levels and postoperative hemoglobin and albumin levels of death group were significantly higher than that of survival group $(\mathrm{P}<0.05)$. Difference of both $\mathrm{Hb}$ and ALB levels before and after operation in death group was significantly higher than that in survival group $(\mathrm{P}<0.05)$. Multivariate logistic regression showed that preoperative NHYA heart function $(\mathrm{OR}=3.692)$, the difference of albumin between pre-operation and post-operation $(\mathrm{OR}=1.229)$ were independently correlated with mortality in AKI patients treated by CRRT after cardiac surgery $(\mathrm{P}<$ $0.05)$.
Conclusion: The mortality of the inpatients suffering from AKI after cardiac surgery and undergoing CRRT is very high. Preoperative NYHA heart function and the difference of albumin between pre-operation and post-operation are the independent risk factors of mortality.

Key Words: Acute kidney injury; Continuous renal replacement therapy; Cardiac surgery; Mortality.

4

\section{Effect of Hydrogen Sulfide and PKC $\beta I I$ in Uremic Accelerated Artherosclerosis Patients}

\author{
Han Li, Su-juan Feng, Shi-xiang Wang* \\ Department of Blood Purification, Beijing Chao-Yang \\ Hospital, Capital Medical University, Nephrology Faculty, \\ Capital Medical University, Beijing, China \\ *Corresponding author: Shi-xiang Wang \\ E-Mail:wxy1988@263.net
}

This work was supported by National Natural Science Foundation of China (81200543), Beijing Natural Science Foundation (7142057) and Beijing Municipal Health Bureau High-level Medical Professionals Promotion Project (2013-3-016).

Objective: Cardiovascular diseases especially atherosclerosis are the major cause of death in chronic hemodialysis (CHD) patients. Hydrogen sulfide $\left(\mathrm{H}_{2} \mathrm{~S}\right)$ plays a protective role in chronic hemodialysis (CHD) patients. We have previously confirmed $\mathrm{H}_{2} \mathrm{~S}$ metabolism abnormalities may contribute to development of uremic accelerated atherosclerosis (UAAS) in chronic hemodialysis patients with diabetic nephropathy. In this study, we try to further explore the relationship between $\mathrm{H}_{2} \mathrm{~S}$ and conventional protein kinase $\mathrm{C} \beta \mathrm{II}$ (cPKC $\left.\beta \mathrm{II}\right)$ in CHD patients with UAAS.

Methods: A total of 20 healthy people, $30 \mathrm{CHD}$ patients without atherosclerosis and 30 chronic hemodialyzed patients with atherosclerosis (CHD+AS) were enrolled if they were more than 18 years of age, had no residual renal function, and had maintained hemodialysis for more than 3 months. CHD patients with atherosclerosis were defined as localized thickening of intima-media thickness (IMT) $\geq 1.2$ $\mathrm{mm}$ that did not uniformly involve the whole wall of carotid artery. Plasma $\mathrm{H}_{2} \mathrm{~S}$ was measured with a sulfide sensitive electrode, and cPKC $\beta$ II membrane translocation was detected by western blot.

Results: There was no significant difference between CHD and $\mathrm{CHD}+\mathrm{AS}$ group in terms of age, sex ratio, dialysis duration, smoking, body mass index (BMI), $\mathrm{K} t / V$, hemoglobulin $(\mathrm{Hb})$, serum creatinine, blood urea nitrogen (BUN), triglyceride (TG), total cholesterol (TC), and etc. Plasma $\mathrm{H}_{2} \mathrm{~S}$ level in CHD patients was significantly lower than the control group, and CHD+AS group was lower than that in CHD patients $(P<0.05)$. cPKC $\beta$ II membrane translocation in $\mathrm{CHD}+\mathrm{AS}$ group increased significantly compared with CHD group $(P<0.05)$. Furthermore, Plasma $\mathrm{H}_{2} \mathrm{~S}$ concentration was negatively correlated with cPKC $\beta$ II membrane translocation in $\mathrm{CHD}+\mathrm{AS}$ patients.

Conclusions: These findings suggest that lower $\mathrm{H}_{2} \mathrm{~S}$ may include in the development of UAAS in CHD patients through regulating the $\mathrm{cPKC} \beta \mathrm{II}$ signaling pathway.

Key Words: Hemodialysis; Uremic accelerated artherosclerosis; Hydrogen sulfide; Protein kinase C $\beta I I$. 


\section{5}

\section{IFNy Inhibit Rat Vascular Smooth Muscle Cells Calcification Induced by High Phosphorus}

Ya-ling Bai, Jin-sheng Xu*, Yue-tong Qian, Jun-xia Zhang, Sheng-lei Zhang, Li-wen Cui

Department of Nephrology, The Fourth Hospital of Hebei Medical University, Shijiazhuang, PR China

${ }^{*}$ Corresponding author: Jin-sheng Xu,

E-Mail: xjs5766@126.com

Objective: To explore the effect of IFN- $\gamma$ on rat vascular smooth muscle cell (VSMCs) calcification.

Methods: VSMCs were obtained from rat aortic and identified by immunocytochemistry, then randomly divided into control group, high phosphorus group, IFN- $\gamma$ intervention group. Calcification staining, calcium content and alkaline phosphatase (ALP) activity were measured and the expression of BMP-2, SMAD1, Cbf $\alpha 1$ mRNA was detected by RT-PCR and the expression of $\mathrm{Cbf} \alpha 1$ protein was detected by western blot after stimulating 3 days.

Results: Compared with the control group, cell calcification, ALP activity and the mRNA expression of Cbfa1, BMP-2, SMAD1 were increased in the high phosphorus group $(\mathrm{P}<0.05)$, while compared with the high phosphorus group, the calcium deposition, ALP activity and the mRNA expression of BMP-2, SMAD1, Runx2 decreased significantly in IFN- $\gamma$ intervention group $(\mathrm{P}<0.05)$.

Conclusion: IFN- $\gamma$ can reduce calcification in vascular smooth muscle cells which may be achieved by inhibiting the transdifferentiation of vascular smooth muscle cells.

Key Words: Vascular smooth muscle cells; Hyperphosphate; Calcification; IFN $\gamma$.

\section{6 \\ Single Nucleotide Polymorphisms in the D-Loop Region of Mitochondrial DNA and Disease-Risk and Outcome of Chronic Kidney Disease \\ Sheng-lei Zhang, Jin-sheng $X_{u}^{*}$, Jun-xia Zhang, Li-wen Cui, Hui-ran Zhang, Qi-yao Yu \\ Department of Nephrology, The Fourth Hospital of Hebei Medical University, Shijiazhuang, PR China \\ ${ }^{*}$ Corresponding author: Jin-sheng Xu \\ E-Mail: xjs5766@126.com}

Objective: To analysis the characteristic of single nucleotide polymorphisms (SNPs) in the displacement loop (D-loop) of the mitochondrial DNA (mtDNA) and investigate the association of disease-risk and outcome of Chronic kidney disease (CKD) with the SNPs in D-loop of the mtDNA, we aimed to seek the biomarker for the development and progression of CKD.

Methods: The D-loop region of mtDNA was sequenced in 88 CKD patients attending the Fourth Hospital of Hebei Medical University between 2002 and 2008. In the 88 CKD patients, 42 were male and 46 were female, with mean age $54.6 \pm 17.1$ years. Blood samples were also collected from age-matched healthy controls. Total DNA was extracted using a Wizard Genomic DNA extraction kit (Promega, Madison, WI, USA) and stored at $-20^{\circ} \mathrm{C}$. PCR was performed using a PCR Master Mix Kit according to the manufacturer's instructions (Promega, Madison, WI), and the PCR products were purified before sequencing. Cyclic Cycle sequencing was performed with the Dye Terminator Cycle Sequencing Ready Reaction Kit (Applied Biosystem, Foster City, CA, USA) and the products were then separated on the ABIPRISM Genetic Analyzer 3100 (Applied Biosystem). Polymorphisms were confirmed by repeated analyses from both strands. The $\chi^{2}$ test was used to analyze dichotomous values, such as the presence or absence of an individual SNP in CKD patients and healthy controls. The kidney survival curve was calculated using the Kaplan-Meier method, and compared with the logrank test. Multivariate survival analysis was performed using a Cox proportional hazards model. All statistical analyses were performed using the SPSS13.0 software (SPSS Company, Chicago, IL, USA). For all the statistical tests, $\mathrm{p}<0.05$ was considered statistically significant.

Results: The sex and age of CKD patients were no significantly differ from those of healthy control $(P>0.05)$. SNPs in reference to GenBank accession AC - 000021 were detected at 98 sites of the 982-bp mitochondria D-Loop region from the CKD patients and the healthy controls. The SNP frequency for the 73G, 146C, 150T, 195C and $16266 \mathrm{C}$ in the CKD patients were higher than those in the health controls, but the SNP frequency for the $16290 \mathrm{~T}$ in CKD patients was lower than that in healthy controls. Smoking habit showed an association with survival rates of kidney in CKD patients when the survival time of kidney in smoking CKD patients was lower than those in nonsmoking CKD patients. The survival rates of kidney in higher body mass index (BMI) CKD patients was higher than those in lower BMI CKD patients. The survival time of kidney in the CKD patients with the rare allele $146 \mathrm{C}$ genotype was significantly shorter than that of CKD patients with the frequent allele 146T. These data demonstrated that smoking and SNP at 146 site were the risk factors for the kidney outcome of CKD patients in the multivariate analysis by the Cox proportional hazards model.

Conclusions: SNPs in the mtDNA D-loop were found to be independent prognostic markers for the disease-risk and the kidney survival time in CKD patients. The analysis of genetic polymorphisms in the D-loop might help to identify CKD patient subgroups at high risk for a disease outcome, thereby helping to refine therapeutic decisions in CKD patients.

Key Words: mtDNA; D-loop; SNP; CKD; Risk; Outcome. 


\section{7}

\section{Application of BioZ.com Noninvasive Hemodynamic Monitoring System for Adjusting Ultra Filtration and Maintaining Stable Hemodynamics in Patients Undergoing Maintenance Hemodialysis}

\author{
Jin-sheng Xu*, Xiao-ling Feng, Jun-xia Zhang, Ya-ling Bai, \\ Sheng-lei Zhang, Dong-xue Zhang, Qi-yao Yu, Lei He \\ Department of Nephrology, The Fourth Hospital of Hebei \\ Medical University, Shijiazhuang, PR China \\ ${ }^{*}$ Corresponding author: Jin-sheng Xu \\ E-Mail: xjs5766@126.com
}

Objective: To explore the clinical significance of adjusting the ultra filtration and maintaining stable hemodynamics by using the BioZ.com noninvasive hemodynamic monitoring system.

Methods: The 41 patients treated with maintenance hemodialysis (MHD) were randomly divided into two groups, the case group and the control group, of which all the patients were measured $\mathrm{CO}, \mathrm{SV}$, LCW, LCWI, MAP and altered the ultra filtration in the case group by the BioZ.com noninvasive hemodynamic monitoring system.

Results: 1) Before dialysis, compared with the control group, the TFC of the case group was statistically significant difference $(P>0.05)$. At the end of the dialysis, the TFC of the the case groups reduced obviously than the control group. 2) Before dialysis, compared with the control group, the CO, SV, LCW, LCWI, MAP of the case group was not statistically significant difference $(P>0.05)$. At the end of the dialysis, the CO, SV, LCW, LCWI (both $P<0.05$ ) of the the case groups dropped as not further as the control group, MAP increased higher $(P<0.05)$. 3) Compared with the control group, the rate of the adverse event of the case group reduced $(P<0.05)$.

Conclusion: The BioZ.com noninvasive hemodynaminc monitoring system could be used to conduct adjusting the ultra filtration in order to maintain stable hemodynamics and improve heart function and decrease the advers events of the maintenance hemodialysis patients.

Key Words: BioZ.com noninvasive hemodynamic monitoring system; filtration; hemodynamics; MHD patients.

\section{8 \\ The Level and Clinical Significance of Red Blood Cell Distribution Width in Maintenance Hemodialysis Patients}

\author{
Bo Shen, Jian-zhou Zou*, Jie Teng, Xiao-qiang Ding, \\ Zhong-hua Liu, Xue-sen Cao \\ Department of Nephrology, Zhongshan Hospital, Fudan \\ University Shanghai Institute of Kidney and Dialysis, \\ Shanghai, China \\ *Corresponding author: Jian-zhou Zou \\ E-Mail zou.jianzhou@zs-hospital.sh.cn
}

Objective: To investigate the level of red blood cell volume distribution width in hemodialysis patients and analyze the clinical significance.
Methods: This cohort study was carried out in 356 HD patients (226 male and 130 female) with mean age of (59.2 \pm 14.5$)$ years and mean dialysis vintage of $(64.3 \pm 42.5)$ months from Jan 2012 to Mar 2010. Medical history were collected and parameters such as hemoglobin, red blood cell distribution width (RDW), serum electrolytes, high sensitivity C-reactive protein (hsCRP), serum albumin, prealbumin, ferritin, transferrin saturation, intact parathyroid hormone (iPTH) and N-terminal brain natriuretic peptide (NT-proBNP) were measured before the mid-week hemodialysis. Serum creatinine and blood urea nitrogen were measured before and after hemodialysis and $\operatorname{spK} t / V$ was calculated. Pearson correlation analysis and multiple linear stepwise regression model were applied to analyze relationship between these clinical parameters and RDW. According to the RDW median of $14.9 \%$, the patients were divided into lower RDW group ( $R D W \leq 14.9 \%$ ) and higher RDW group (RDW $>14.9 \%$ ) in the 24 months follow-up. Survival analysis was performed with Kaplan Meier survival curves between the higher RDW group and the lower RDW group. Cox proportional hazards model was used to explore independent predictors related to prognosis.

Results: The mean level of RDW was $15.14 \pm 1.22(13.0 \sim 20.4) \%$ (median 14.9\%) in all patients, of which 241 (67.7\%) were abnormal. RDW in male group was significantly lower than that in female group $[(15.03 \pm 1.11) \%$ vs. $(15.33 \pm 1.39) \%, P<0.05]$. Pearson correlation analysis showed that RDW was positively correlated with age $(\mathrm{r}=$ $0.269, P<0.001)$, dialysis vintage $(\mathrm{r}=0.116, P=0.033)$, EPO dose $(\mathrm{r}=0.250, P<0.001), \mathrm{EPO} / \mathrm{Hb}$ ratio $(\mathrm{r}=0.214, P<0.001)$, hsCRP ( $\mathrm{r}=0.335, P \leq 0.001)$ and NT-proBNP $(\mathrm{r}=0.133, P=0.027)$. RDW was negatively correlated with serum albumin $(\mathrm{r}=-0.241, P<0.001)$ and pre-albumin $(\mathrm{r}=-0.334, P<0.001)$. Multivariate linear regression model showed that RDW was significantly associated with serum albumin and EPO dose $(P<0.01)$. In curve fitting estimation, the quadratic equation had the goodness of fitting with statistical significance (R square $=0.093, P<0.001$ ). A total of 42 cases died during the follow-up period. 12 cases $(6.7 \%)$ in the lower RDW group and 30 cases $(16.9 \%)$ died in the higher RDW group. The causes of death in the lower RDW group were 5 cardiac death (3 sudden cardiac death, 2 heart failure), 4 cerebrovascular accident, 2 serious infection and 1 malignant tumor. The causes of death in higher RDW group were 12 cardiac death ( 6 sudden cardiac death, 6 heart failure), 5 cerebrovascular accident, 5 serious infection, 4 malignant tumor and 4 other reasons. Kaplan Meier survival curve analysis showed that the lower RDW group had a significantly higher survival rate than higher RDW group (Log-rank $=12.124, P<0.001)$. Cox regression model of single factor analysis showed the risk factors related to the death endpoint were age, hemoglobin, serum albumin, red blood cell volume distribution width, intact parathyroid hormone levels $(P<0.05)$. Multiple factors analysis with Cox regression model showed that the red blood cell distribution width $(\mathrm{HR}=1.286, P=0.044)$, serum albumin $(\mathrm{HR}=$ $0.951, P=0.010)$, age $(\mathrm{HR}=1.042, P=0.005)$ were the independent predictors of death in maintenance hemodialysis patients.

Conclusions: Increased RDW was common in hemodialysis patients, which might be related to age, hemoglobin, dialysis vintage, nutrition status, inflammation, heart disease, EPO dose and EPO response. The increased RDW was significantly associated with poor outcome of maintenance hemodialysis patients. RDW was the independent predictor of clinical outcome in hemodialysis patients. Further studies were required to explore the mechanism between RDW and the prognosis in hemodialysis patients.

Key Words: Red blood cell distribution width; Hemodialysis; Cohort Study; Factor Analysis; Prognosis. 


\section{9}

\section{Beneficial Effects of Magnesium on Hyperphosphate-Induced Calcification in Rat Vascular Smooth Muscle Cells}

Jin-sheng $X u^{*}$, Rong-fang Zhu, Jing-jing Jin, Ya-ling Bai, Jun-xia Zhang, Li-wen Cui, Wei Zhou

Department of Nephrology, The Fourth Hospital of Hebei Medical University, Shijiazhuang, PR China

${ }^{*}$ Corresponding author: Jin-sheng Xu

E-Mail: xjs5766@126.com

Objective: Vascular calcification is recognized as a common complication in patients suffering from chronic kidney disease. Many factors, particularly hyperphosphatemia, have been shown associated with the high prevalence of vascular calcification. Recently, the beneficial effects of magnesium on vascular calcification have been concerned. The present study aimed to investigate the role of magnesium on calcification induced by $\beta$-glycerophosphate $(\beta-\mathrm{GP})$ in rat vascular smooth muscle cells.

Methods: In vivo, Male SD rats were made uremic with vascular calcification by feeding adenine suspension and high phosphorous diet in presence of magnesium $(0.02 \%, 0.05 \%, 0.15 \%)$ for 15 weeks. In vitro, Primary rat vascular smooth muscle cells (VSMCs) were exposed to $10 \mathrm{mM} \beta$-GP in medium with or without magnesium (1.0, 2.0, $3.0 \mathrm{mM}$ ) or 2-aminoethoxy-diphenylborate (2-APB; an inhibitor of magnesium transport) for 14 days. Calcium deposition was determined by von Kossa staining, Alizarin red staining and quantification of calcium. The aortic pulse wave velocity (PWV) was observed, and core-binding factor $\alpha-1$ (Cbf $\alpha 1)$ in aortic was measured by immunhistochemistry. In vitro, the expression levels of Cbf $\alpha$, matrix Gla protein (MGP) and osteopontin (OPN) were determined by reverse transcription-polymerase chain reaction (RT-PCR) or western blot analysis, following incubation for $0,3,6,10$ and 14 days with the different media, and alkaline phosphatase (ALP) activity were measured by enzyme-linked immunosorbent assay (ELISA).

Results: In vivo, high phosphorous administration to uremic rats with control magnesium resulted in significant calcium deposition in the aorta, increased aortic PWV and Cbf $\alpha 1$ expression. High phosphorous-treated uremic rats with high magnesium inhibited these phenomena, but low magnesium aggravated these effects. In vitro, higher magnesium may reduce VSMC calcification and ALP activity significantly compared with the calcification medium, and the inhibitory effect was in dose-dependent manner. The magnesium-induced changes in the VSMCs included a downregulation of Cbf $\alpha 1$ expression which was induced by $\beta$-GP after 3 days incubation, an effect that was gradually enhanced over the 14 days period. By contrast, magnesium produced notable increases in MGP and OPN expression levels, with an opposite pattern to that observed in the Cbf $\alpha 1$ expression levels. However, the addition of 2-APB appeared to inhibit the protective effect of magnesium on the VSMCs.

Conclusions: Magnesium was able to effectively reduce $\beta$-GPinduced calcification in rat VSMCs in dose-dependent manner. The mechanism may be attributed to the regulation of the expression levels of calcification-associated factors in a time-dependent manner.

Key Words: Vascular smooth muscle cells; Hyperphosphate; Calcification; Magnesium.
10

\section{Effect of Combined Culture Methods on Bacterial Colony Count in Hemodialysis Water and Dialysate}

\author{
Hong Cai, ${ }^{1,2}$ Wei-ming Zhang 1,2**, Hai-hui Yang ${ }^{3}$, \\ Yong-mei Wang ${ }^{1,2}$, Bin Zhang ${ }^{1,2}$, Rong Jiang ${ }^{1,2}$, \\ Chun-mei Ying ${ }^{3}$, Yu-cheng Yan ${ }^{1,2}$. Zhao-hui Ni ${ }^{1,2}$, \\ Jia-qi Qian ${ }^{1,2}$ \\ 'Department of Nephrology, Ren Ji Hospital, School \\ of Medicine, Shanghai Jiao Tong University, Shanghai \\ China; ${ }^{2}$ Department of Nephrology, South Campus, \\ Renji Hospital, School of Medicine, Shanghai Jiao Tong \\ University, Shanghai, China; ${ }^{3}$ Department of Clinical \\ Laboratory, Renji Hospital, School of Medicine, Shanghai \\ Jiaotong University, Shanghai, China \\ ${ }^{*}$ Corresponding author: Wei-ming Zhang \\ E-Mail:weimingzh1965@163.com
}

This study was sponsored by the South Campus, Shanghai Renji Hospital, (Multi-discipline collaboration project, 2014 MDT 02).

Objective: To compare the detection ratio of bacterial colony count in dialysis water, dialysate and raw water with different media and different incubation conditions, searching for suitable methods to detect bacteria from dialysis water.

Methods: Between Jan 2012 and Dec 2012, 176 samples of hemodialysis water and dialysate, 88 samples of raw water were collected by hemodialysis unit, Renji hospital. Samples were inoculated in duplicate on spread plates with Blood agar, Reasoner's 2A (R2A), Tryptone glucose extract agar (TGEA) respectively, at different temperature and time. After incubation, the numbers of colonies were quantified.

Results: The bacterial colony counts in dialysis water, dialysate and raw water were the lowest in blood agar and the difference had statistical significance when it was compared with that of R2A or TGEA culture media $(\mathrm{P}<0.01)$. The detection rate of dialysis water and dialysate were $61.9 \%$ in R2A at $20^{\circ} \mathrm{C}$ for 168 hours. According to the standard of the association for the advancement of medical instrumentation (AAMI), the detection rate had no difference among these media with different conditions. Combined with medium of R2A and TGEA, the detection rate of bacteria was $77.6 \%$ at $20^{\circ} \mathrm{C}$ for 168 hours and the bacteria detection rate was significantly increased than that in culture R2A or TGEA respectively under the same conditions $(77.6 \%$ vs. $61.9 \%, 50.6 \%$, P value was 0.003 and 0.001 ).

Conclusions: The method of R2A or TGEA agar culture can improve the detection rate of bacterial colony count compared with the method of blood agar. The method of R2A combined with TGEA at $20^{\circ} \mathrm{C}$ for 168 hours can improve the detection rate of bacteria in dialysis water and dialysate.

Key Words: Hemodialysis; Dialysate; Culture techniques; Bacterial culture; Dialysis water; Culture medium. 


\section{1}

\section{The Impacts of Ultrafiltration Rate and Cardiac Function on the Outcomes among IDH Patients: A 5-Year Prospective Cohort Study}

\author{
Jin-bo Yu, Jian-zhou Zou, Zhong-hua Liu, Bo Shen, \\ Jie Teng, Xiao-qiang Ding*
}

Department of Nephrology, Zhongshan Hospital, Shanghai Medical College, Fudan University, Shanghai Institute of

Kidney and Dialysis, China

${ }^{*}$ Corresponding author: Xiao-qiang Ding

E-Mail: ding.xiaoqiang@zs-hospital.sh.cn

Objective: To acknowledge the relationships between intradialytic-hypotension (IDH) and ultrafiltration rate, as well as cardiac function for the prevention and treatment of IDH.

Methods: 117 IDH-prone patients (IDH $\geq 1 / 10$ hypotensive events/3 months) were recruited during Jan. 2009 to Dec. 2009. Intradialytic blood pressure was monitored during a 3-month period. IDH was defined as an event characterized by a sudden drop in systolic BP more than $20 \mathrm{~mm} \mathrm{Hg}$ or in mean artery pressure (MAP) more than $10 \mathrm{~mm} \mathrm{Hg}$ associated with clinical events and need for interventions. Patients were then divided into 2 groups according to the ultrafiltration volume/body weight 5\%. Dialysis-related information was collected. Kaplan-Meier method, log-rank test and Cox regression analyses were performed to examine the association between ultrafiltration rate, as well as cardiac function and survival in IDH-prone patients, using a follow-up through 31 Dec 2014.

Results: During 5-year follow-up, 30 patients died, with a mortality rate $5.6 \%$ per year. There was no difference of overall mortality rate between 2 groups using Kaplan-Meire survival curve $(P=$ 0.878). The multivariate Cox regression model indicated that high ultrafiltration rate increased the risk of death $(H R=1.169,95 \% C I$ : $1.012 \sim 1.351, P=0.034)$. We compared the cardiac function at baseline and the end of the study by echocardiography. The ejection fraction in high ultrafiltration-rate group decreased significantly. In high ultrafiltration-rate group, multivariate Cox regression model indicated that high ejection fraction reduced 5-year mortality $(H R=0.864,95 \%$ $C I$ : 0.767 0.974, $P=0.016)$. While high serum NT-proBNP increased 5-year mortality $(H R=8.568,95 \% C I: 2.079 \sim 35.319, P=0.003)$. In low ultrafiltration-rate group, multivariate Cox regression model indicated high ultrafiltration rate increased the risk of death $(H R=$ $1.273,95 \%$ CI: $1.002 \sim 1.617, P=0.048$ ).

Conclusion: High ultrafiltration rate is the leading risk factor for poor outcome in IDH-prone patients. Some IDH-prone patients have pretty good cardiac function, but might develop cardiac insufficiency due to long-term over-ultrafiltration.

Key Words: Intradialytic hypotension; Cardiac function; Ultrafiltration rate; Outcome.
12

\section{Effect of Continuous Quality Improvement on the Incidence of Peritonitis in Chronic Peritoneal Dialysis Patients}

\author{
lei Ran, Qiong-zhen Lin*, Wang Xiu-fen, Fu-yan Guo, \\ Wei Wei \\ Renal Division, Third hospital, Hebei Medical University, \\ Shijiazhuang, Hebei, China \\ * Corresponding author: Qiong-zhen Lin \\ E-Mail: qzlin@vip.sina.com
}

Objective: We investigated the role of continuous quality improvement (CQI) initiative in decreasing the incidence of peritonitis in chronic peritoneal dialysis patients.

Method: In a retrospective study, 2 yeas of data from 2013 before CQI and 2014 after CQI were analyzed. Four-step program for CQI (i.e. plan-do-check-act) was implemented to decrease the incidence of peritoneal dialysis related peritonitis. The incidence and the characteristics of peritonitis were calculated and analyzed before and after CQI.

Result: There were 28 episodes of peritonitis in 22 out of total 99 PD patients in 2013. 17 cases with peritonitis occurred for the fist time and 11 of them $(64.7 \%)$ occurred in their first 5 months after PD initiate. Culture-positive was in 12 episodes (6 episodes of $S$ aureus and 2 episodes of Escherichia coli.). The rate of culture-positive peritonitis was $43.9 \%$. 3 patients with peritonitis were drop out from PD ( 2 patients transferred to HD, 1 patient died.) The whole year peritonitis rate was 1 episode every 35.2 months in 2013 before CQI. There were 12 episodes of peritonitis in 10 patients out of 101 patients after CQI in 2014. There were 9 episodes of peritonitis occurred for the first time and 3 of them (33.3\%) occurred in their first 4 months after PD initiate. The rate of culture-positive peritonitis were increased to $66.7 \%$. In the 8 culture-positive episodes, 3 were $S$. aureus and 5 were Escherichia coli. The whole year peritonitis rate decreased to 1 episode every 108.5 months in 2014 after CQI. 1 patients dropped out and transftered to HD in 2014.

Conclusion: Integration of a CQI process into a PD program can significantly decrease the incidence of peritoneal dialysis related peritonitis in our PD centre.

Key Words: Peritoneal dialysis, Peritonitis, Continual quality improvement. 


\section{3 \\ Evaluation of Safety and Efficacy of Polyether Membrane Dialyser in Hemodiafiltration}

\author{
Zhong-hua Liu'1,2, Jian-zhou Zou'1,2, Wei-ming Zhang ${ }^{3}$, \\ Bo Shen ${ }^{1,2}$, Wen-lv Lv ${ }^{1,2}$, Xue-sen Cao ${ }^{1,2}$, Lin Hu ${ }^{1,2}$, \\ Yu-cheng Yan ${ }^{3}$, Yong-mei Wang ${ }^{3}$, Hong Cai ${ }^{3}$, Ren-hua Lu ${ }^{3}$, \\ Yan Fang ${ }^{3}$, Hong Jiang ${ }^{3}$, Xiao-qiang Ding ${ }^{1,2, *}$ \\ ${ }^{1}$ Division of Nephrology, Zhongshan Hospital, Fudan \\ University, Shanghai, China; ${ }^{2}$ Shanghai Institute of Kidney \\ and Dialysis, Shanghai, China; ${ }^{3}$ Division of Nephrology, \\ Renji Hospital, Shanghai Jiaotong University, Shanghai, \\ China \\ *Corresponding author: Xiao-qiang Ding \\ E-Mail: ding.xiaoqiang@zs-hospital.sh.cn
}

Objective: To evaluate the safety and efficacy of polyether membrane dialyser (PES18HDF) in hemodiafiltration.

Methods: Maintenance hemodialysis patients were randomized to two groups: polyether membrane dialyser (PES18HDF) and polysulfone membrane dialyser (Diacap ${ }^{\circledR} \alpha$ Polysulfone ${ }^{+} \mathrm{HiFlo}$ ). The safety and efficacy indicators were observed.

Results: 76 patients were enrolled in the trial. There were 38 cases in each group. $\beta_{2}-\mathrm{MG}$ in experimental group decreased $79.67 \pm$ $3.39 \%$, which was significantly higher than that of the control group of $75.52 \pm 5.43 \%(\mathrm{p}=0.0003)$. Urea clearance rate was $241.56 \pm$ $24.25 \mathrm{ml} / \mathrm{min}$, significantly higher than that in the control group for $222.36 \pm 30.18 \mathrm{ml} / \mathrm{min}(\mathrm{p}=0.0042)$. Creatinine clearance rate of the two groups was $163.87 \pm 29.84$ and $154.84 \pm 32.34 \mathrm{ml} / \mathrm{min}$, with no significant difference $(\mathrm{p}=0.2221)$. The ultrafiltration coefficient of the experimental group was $35.80 \pm 8.38 \mathrm{ml} / \mathrm{mm} \mathrm{Hg} / \mathrm{h}$, which was significantly higher than that of the control group for $31.55 \pm$ $7.45 \mathrm{ml} / \mathrm{mm} \mathrm{Hg} / \mathrm{h}(\mathrm{p}=0.0248)$. Adverse events occurred 5 times in the experimental (occurrence rate 13.16\%), and once in the control group (occurrence rate $2.63 \%$ ). But there was no significant difference between the two groups $(\mathrm{p}=0.2)$.

Conclusion: Polyether membrane used in this research was confirmed to have better urea and $\beta_{2}$-MG removal with good safety. The long-term clinical consequences of better uremic toxin removal should be prospectively assessed.

Key Words: Hemodiafiltration; Dialyser; Polyether membrane; $\beta_{2}$-microglobulin.

\section{4}

\section{Molecular Basis of the Brain-Type Dialysis Disequilibrium Syndrome: Altered Aquaporin 1,5 and Urea Transporter Expression in the Brain}

\author{
Zhen-wei Shi, Yi Liu, Wei Liu, Yan Xu, Xiao-Iu Li, \\ Qing-yang Liu*, Zhi-gang Wang \\ Renal Department, China Meitan General Hospital, Beijing, \\ China \\ * Corresponding author: Qing-yang Liu \\ E-Mail: Ilqq2003yy@aliyun.com
}

Objectives: To discuss the expression levels of aquaporin 1, 5 and urea transportproteinB1 in the braintissue of mice with chronic renal failure, which maybe the molecular basis of brain-type dialysis disequilibrium syndrome.

Methods: We selected BALB/c mice and divided them into three groups randomly: the normal group, the sham operation group and the chronic renal failure group using renal cortical diathermy. Five mice in each group were sacrificed in 10 days, 40 days and 70 days after modeling. We got serum specimens, brains and kidneys. Serum creatinine, blood urea nitrogen, kidney tissue pathology were detected, and western blotting were used to determine the AQPs and urea transportproteinB1 expression.

Results: The renal histopathologic findings shown that glomerular proliferation and sclerosis, kidney tubules atrophy and fibrosis in the chronic renal failure group in 10,40,70 days after modeling. The serum creatinines respectively are $841.80 \pm 336.93 \mathrm{umol} / \mathrm{L}, 1885.17 \pm$ $689.49 \mathrm{umol} / \mathrm{L}, 1276.56 \pm 496.09 \mathrm{umol} / \mathrm{L}$ in the chronic renal failure group which increased significantly, compared with the normal group and the sham group $(\mathrm{F}=26.768, \mathrm{P}<0.007)$ in 10 days, 40 days and 70 days after modeling. The results of western blotting showed that the brain AQP1 expression in the model group increased $46.67 \%$ $(1.10 \pm 0.05$ vs. $0.75 \pm 0.05, \mathrm{t}=0.122, \mathrm{P}<0.001)$ in 10 days after modeling, $28.98 \%(0.89 \pm 0.02$ vs. $0.69 \pm 0.04, \mathrm{t}=4.926, \mathrm{P}<0.001)$ in 40 days after modeling, and 30.26\% (0.99 \pm 0.07 vs. $0.76 \pm 0.05, t=$ $8.471, \mathrm{P}<0.001)$ in 70 days after modeling, compared with the control groups; The brain AQP5 expression in the model group increased $41.09 \%(1.03 \pm 0.03$ vs. $0.73 \pm 0.02, \mathrm{t}=0.012, \mathrm{P}<0.001)$ in 10 days after modeling, $20.83 \%(0.87 \pm 0.03$ vs. $0.72 \pm 0.04, \mathrm{t}=0.857, \mathrm{P}<$ $0.01)$ in 40 days after modeling, and $45.67 \%(1.18 \pm 0.09$ vs. $0.81 \pm$ $0.04, \mathrm{t}=3.352, \mathrm{P}<0.001)$ in 70 days after modeling. The brain urea transport protein $\mathrm{B} 1$ expression in the model group reduced significantly compared with the normal group and the sham operation group in 10 days, 40 days and 70 days after modeling.

Conclusions: The combination of increased expression of AQP1,5 and decreased expression of urea transport-protein B1 in the brain tissue of the chronic renal failure mice may be the molecular basis of brain-type dialysis disequilibrium syndrome. The clearance delay of urea happened during the rapid hemodialysis because of the low expression of urea transport-protein $\mathrm{B} 1$, and then concentration gradient of urea formed between brain and blood, this created osmotic driving force that promoted water into the brain tissue by the overmuch aquaporins and subsequent encephaledema.

Key Words: Hemodialysis; Dialysis disequilibrium syndrome; Aquaporin; Urea transporter. 


\section{5}

\section{Short and Long-Term Efficacy of Total Parathyroidectomy with Forearm Autograft for Secondary Hyperparathyroidism in $\mathbf{8 0}$ Dialysis Patients}

\author{
Jun Ji, Hong-wei Zhang, Jing Lin, Shao-min Gong, \\ Li Wang, Pan Lin, Yi-mei Wang, Chun-feng Liu, Bo Shen, \\ Zhong-hua Liu, Xiao-qiang Ding* \\ Department of Nephrology, Zhongshan Hospital, Fudan \\ University, Shanghai Institute for Kidney and Dialysis, \\ Shanghai, China \\ ${ }^{*}$ Corresponding author: Xiao-qiang Ding \\ E-Mail:ding.xiaoqiang@zs-hospital.sh.cn
}

Objective: To evaluate the short- and long-term impact of total parathyroidectomy with forearm autograft (TPTX+FAT) in the dialysis patients with secondary hyperparathyroidism.

Methods: We performed a retrospective review of parathyroidectomies with forearm autograft in the dialysis patients performed in Zhongshan Hospital of Fudan University between January 2009 and March 2015. The outcomes were compared on patient demographics, exams consisting of ultrasonic cardiogram (UCG) and pulse wave velocity (PWV), and blood parameters consisting of calcium $(\mathrm{Ca})$, phosphorus (P), and intact parathyroid hormone (iPTH) pre- and postoperatively. Clinical indications for parathyroidectomy for renal hyperparathyroidism with maintenance hemodialysis were a persistent high parathyroid hormone level (PTH $>800 \mathrm{pg} / \mathrm{ml}$ ), uncontrollable hypercalcemia or hyperphosphatemia, with severe symptoms such as ostalgia, myalgia and skin itch, and detection of enlarged parathyroid glands by image diagnosis (diameter of the largest gland $>10 \mathrm{~mm}$ by ultrasonography and concentration of parathyroid glands by technetium $99 \mathrm{~m}$ subtraction scintigraphy, the abscised glandular tissue was sliced into pieces $(1 \times 1 \times 1 \mathrm{~mm})$, then autotransplanted into the forearm muscle without arteriovenous fistula for hemodialysis. The criterion of relapse was elevated iPTH level $(>500 \mathrm{pg} / \mathrm{ml})$ with severe symptoms.

Results: 80 patients (46 males and 34 females, average age $51.4 \pm 12.5$ years, 65 hemodialysis with average HD time before operation $109 \pm 50.6$ months; and 15 peritoneal dialysis with average PD time before operation $70 \pm 20.4$ months) underwent TPTX+FAT were retrieved from our database. The success rate was $100 \%$. There was no death in perioperative period. Postoperatively, 72 patients $(90 \%)$ with hypocalcemia, including 8 patients $(10 \%)$ with low calcium tic were supplemented with calcium and cholecalciferol. And 4 Patients $(5 \%)$ were suffered from hypothyroidism were supplemented with L-Thyroxine. 4 patients relapsed after 6 months, who were controlled by medications and parathyroidectomy respectively. Postoperatively, the symptoms such as ostalgia, myalgia and skin itch were all relieved in 1 month. And serum $\mathrm{Ca}, \mathrm{P}$, and $\mathrm{iPTH}$ in 1st week, 1st month, 3rd months, 6th months, 1st year and 3rd years after operation, significantly improved compared with those before operation (all $\mathrm{p}<0.05$, shown in table 1). 20 patients $(50 \%)$ with cardiovascular calcification were followed up and the UCG and PWV did not differ significantly pre- and postoperatively.

Conclusions: We conclude that parathyroidectomy and forearm autograft is a safe treatment for renal hyperparathyroidism in dialysis patients. It is effective on controlling the symptoms, serum $\mathrm{Ca}, \mathrm{P}$, and $\mathrm{PTH}$, but ineffective in treating cardiovascular calcification that already exists before operation.

Key Words: Parathyroidectomy; Secondary hyperparathyroidism; Dialysis; Cardiovascular calcification.

\section{6 \\ Cross-District Hemodialysis Trends in Maintenance Hemodialysis Patients in Beijing Area}

Bing-han Li, Li Zuo

Department of Nephrology, Peking University First Hospital, Nephrology Institute, Peking University, Key Laboratory for Nephrology Diseases, National Ministry of Health, Key Laboratory for Prevention of Nephrology Diseases, National Ministry of Education, Beijing, China Corresponding author: Li Zuo*

E-Mail: zuoli@bjmu.edu.cn

Objective: The aim of this study was to examine the cross-district dialysis trends in maintenance hemodialysis patients in recent years in Beijing area.

Methods: The data of all patients were obtained from the Beijing Hemodialysis Quality Control and Improvement Center (BJHDQCIC) from 2007 to 2013. Patients with unknown residential address or unclear dialysis unit location were excluded. Crossdistrict dialysis trends were evaluated by comparing residential address and hemodialysis unit location. The specific workload of each district was assessed by two ratios: the ratio of dialyzed patients and patients resided in this district, and the ratio of dialyzed patients and hemodialysis equipment number in this district.

Results: A total of 9591 patients met the inclusion criteria, 34.28 percent patients received cross-district dialysis (hemodialysis unit and habitation were not in the same district). In the patients lived in inner suburb, $44.62 \%$ were getting regular dialysis in urban, while in outer suburb, this proportion was $12.23 \%$. Patients from suburb may increase workload of hemodialysis units in urban. In the four urban

Table 1. Comparison of blood parameters pre- and postoperatively (for Abstract 15)

\begin{tabular}{|c|c|c|c|c|c|c|c|}
\hline & Pre-operation & 1st week & 1st month & 3rd months & 6th months & 1st year & 3rd year \\
\hline Serum calcium (mmol/l) & $2.57 \pm 0.22$ & $1.53 \pm 0.38$ & $1.78 \pm 0.23$ & $1.93 \pm 0.35$ & $2.0 \pm 0.39$ & $2.11 \pm 0.40$ & $2.21 \pm 0.43$ \\
\hline Serum phosphate $(\mathrm{mmol} / \mathrm{l})$ & $2.20 \pm 0.59$ & $1.43 \pm 0.69$ & $1.43 \pm 0.60$ & $1.56 \pm 0.71$ & $1.65 \pm 0.80$ & $1.64 \pm 0.89$ & $1.73 \pm 0.99$ \\
\hline iPTH (pg/ml) & $1,766.4 \pm 344.2$ & $155.3 \pm 187.2$ & $133 \pm 190.7$ & $145 \pm 188.4$ & $167 \pm 223.9$ & $177 \pm 395.6$ & $198 \pm 412.8$ \\
\hline Patients & 80 & 80 & 80 & 76 & 73 & 66 & 56 \\
\hline
\end{tabular}

Annual Congress of the Chinese Blood 
district, the ratios of dialyzed patients and lived patients were 2.37 (HaiDian), 1.61 (XiCheng), 1.29 (ChaoYang) and 0.93 (DongCheng). While the ratios of dialyzed patients and equipment number were 3.19 (HaiDian), 4.57 (XiCheng), 3.61 (ChaoYang) and 3.06 (DongCheng).

Conclusion: More than one-third maintenance hemodialysis patients was getting cross-district dialysis. Most of cross-district pattern was from suburb to urban, which increased workload of hemodialysis units in urban.

Key Words: Maintenance hemodialysis; Cross-district hemodialysis; Renal data register system.

17

\section{The Characteristics and Influencing Factors of Blood Pressure in MHD Patients during Dialysis Day}

\author{
Oian Wang, Ying Li*, Bao-xing Wang, Yan-qing Chi, \\ Jian Gao \\ The Third Hospital of Hebei Medical University, Hebei, China \\ * Corresponding author: Ying Li \\ E-Mail: liyinghebei@126.com
}

Objective: The purpose of this study is aimed to observe the characteristics and circadian rhythm of blood pressure during dialysis day, and explore the influencing factors, then control blood pressure and provide theoretical basis for avoiding cardial vascular disease (CVD).

Methods: 1) object and date: We enrolled 41 MHD patients into the study, who is conformed to the condition in the third hospital of Hebei medical university. 24-hour ambulatory blood pressure monitor was used to monitor the blood pressure during dialysis day, in order to get parameters, including 24-hour systolic blood pressure, 24-hour diastolic blood pressure, decline in nocturnal systolic and diastolic blood pressure and so on. All patients were applied body composition monitor (BCM) for monitoring volume status before hemodialysis, in order to get TBW, ECW, ICW, ECW/ICW, ECW/TBW, OH and other volume load index. General clinical data, complications, dialysis solutions, antihypertensive drugs and laboratory parameters before or after a month were recorded. 2) Group and statistical analyses: Two groups were identified with their ambulatory blood pressure: the blood pressure controlled group and blood pressure uncontrolled group. Three groups were identified with range of decline rate of nocturnal systolic blood pressure: dipper group, non-dipper group, anti-dipper group. Patients' general statistical data, laboratory parameters and volume load index of the two groups were compared, then multivariate stepwise regression was performed respectively between the 24-hour systolic blood pressure, 24-hour diastolic blood pressure, decline in nocturnal systolic blood pressure, decline in nocturnal diastolic blood pressure and above observations indicators. All of the analyses were performed using SPSS software 19.0 and $P<0.05$ was considered indicative of statistically significant differences.

Results: 1) During dialysis day the blood pressure controlled group included 9 patients $(22.0 \%)$, and blood pressure uncontrolled group included 32 patients $(78.0 \%)$. The dipper group included 6 patients $(14.6 \%)$, the non-dipper group included 23 patients $(56.1 \%)$, and the anti-dipper group included 12 patients (29.3\%). 2) The level of parathyroid hormone in blood pressure uncontrolled group was higher than that of the controlled group $(255.13 \pm 21.98$ vs. $134.08 \pm 19.19$,
$P=0.005$ ); and the gender composition ratio, age, duration of dialysis, IDWG, diabetes mellitus, EPO dosage, hemoglobin, serum albumin, pre-dialysis serum creatinine, pre-dialysis serum urea, serum calcium, serum phosphorus, dialysis adequacy (Kt/v, URR) and volume load index, including $\mathrm{OH}, \mathrm{ECW} \%, \mathrm{ICW} \%$, ECW/ICW, ECW/TBW were not significantly differed between the blood pressure controlled group and blood pressure uncontrolled group $(P>0.05)$. The level of parathyroid hormone in the dipper group was lower than that of the other two groups $(120.28 \pm 15.25 \mathrm{pg} / \mathrm{ml}$ vs. $238.75 \pm 28.31 \mathrm{pg} / \mathrm{ml}$ vs. $263.16 \pm$ $28.76 \mathrm{pg} / \mathrm{ml}, P=0.018)$, and the other indicators were not significantly differed between each other $(P>0.05)$. 3) Multivariate stepwise regression analysis were respectively applied, 24-hour systolic blood pressure, 24-hour diastolic blood pressure, decline in nocturnal systolic blood pressure, decline in nocturnal diastolic blood pressure as the dependent variable, and the above indicators as independent variables. The results showed that 24 -hour systolic blood pressure was positively correlated with ECW/ICW ( $\beta=72.282, P=0.007)$; 24-hour diastolic blood pressure was negatively correlated with age $(\beta=-0.631, P<$ $0.001)$; decline in nocturnal systolic blood pressure was positively correlated with hemoglobin $(\beta=0.183, P=0.024)$; decline in nocturnal diastolic blood pressure was positively correlated with hemoglobin $(\beta=1.358, P=0.001)$, serum albumin $(\beta=0.266, P=0.003)$, and it was negatively correlated with serum calcium $(\beta=-26.822, P=0.002)$.

Conclusions: 1) MHD patients have a prevalent hypertension and circadian rhythm abnormalities, and the proportion of the decline in nocturnal blood pressure are decreased. Blood pressure rhythm is mainly characterized by non-dipper, even anti-dipper. 2) The blood pressure level and circadian rhythm during dialysis day are influenced by overhydration, parathyroid hormone, age, and so on. The heavier the capacity and the higher parathyroid hormone levels, then the higher blood pressure, the lower rate of nocturnal blood pressure decline rate, the higher proportion of non-dipper and the proportion of anti-dipper rhythm. 3) The change and influencing factors during hemodialysis in MHD patients were complicated, more studies are needed in this field.

Key Words: Maintenance hemodialysis; Dialysis day; Ambulatory blood pressure; Circadian rhythm; Influencing factors.

\section{8}

\section{Clinical Research of Serum ET-1 and NO at the Early Stage of Establishing Autologous Arteriovenous Fistula}

\author{
Hong-ye Dong, Fang Wei, Li-hua Wang, Ai-li Jiang* \\ Department of Kidney Disease and Blood Purification \\ Treatment, Institute of Urology and Key Laboratory \\ of Tianjin, 2nd Affiliated Hospital of Tianjin Medical \\ University, Tianjin, 300211, China \\ Corresponding author: Ai-li Jiang \\ E-Mail: 75455958@qq.com
}

Objective: Hemodialysis is an effective way of replacement therapy for end-stage renal disease (ESRD) patients. Good vascular access is the key to ensure the smoothly progress of hemodialysis. Autogenous arteriovenous fistula (autologous arteriovenous fistula, AVF) is the best choice for long-term hemodialysis patients. Mature autogenous arteriovenous fistula refers to that shoud have minimal 
risks of extravasation during fistula puncture, and it provide adequate blood flow during the entire hemodialysis process. At present, there is no uniform standard for mature autogenous arteriovenous fistula. $\mathrm{K} / \mathrm{DOQI}$ guidelines pointed out that fistula can be used at least one month after surgery and the better time may be 6-8 weeks after surgery; K/DOQI have refered that fistula maturation should follow three "6" principle which included fistula blood flow $>600 \mathrm{ml} /$ min, arterial of venous vessel diameter $>0.6 \mathrm{~cm}$, subcutaneous depth $<0.6 \mathrm{~cm}$, and clearly visible blood vessel boundary [1]. But those are only the views which lack of evidence support. The Working Group failed to reach agreement about the ideal fistula maturation criteria. Several articles about AVF which use color Doppler ultrasonography in measurement of diameter and blood flow, found that blood flow usually increase 1-4 weeks after establishment of fistula. Authors of these articles thought that the blood flow rate tend to be stabilized one month after surgery, so simply use blood flow rate and blood vessel diameter as a standard to evaluate the maturation of fistula is not better enough. This research monitored the serum concentration of endothelin-1 and nitric oxide evaluate the vascular endothelial injury repair process and contrasted the color doppler ultrasound examination results within the first four weeks postoperation. This research aim to explore the changes of systemic hemodynamics at the early stage of establishing autologous arteriovenous fistula and to investigate the optimal puncture time.

Materials and Methods: This study selected 33 patients with end-stage renal disease from blood purification center of our hospital and then followed up. The patient initial stat renal replacement therapy and long-term path were autogenous arteriovenous fistula, while excluding cancer, autoimmune disease, sever infection or acute infection, congenital heart disease, valvular heart disease, severe arrhythmia, heart disease, exhaustion, life expectancy of less than six months, severe anemia (HGB $<60 \mathrm{~g} / \mathrm{l}$ ), blood diseases. All of the patientts were taken fasting venous blood in the morning respectively on day of surgery and 1,2,3,4 weeks after operation by routinely method to test serum glucose (Glu), lipid, renal function, albumin, serum calcium and phosphorus. Nitric oxide (NO) and endothelin-1 (ET-1) were measured by ELISA. The general condition of the patients were recorded. Two-dimensional color Doppler examination were used to measure the diameters of anastomosis, arterial trunk and venous 5 times respectively 4 weeks before and after operation. Blood flow rate were automatically calculated by the built-in software of ultrasound system and the peak flow were recorded. Application of statistical package SPSS 17.0 to statistical analysis of all data, $\mathrm{P}<$ 0.05 was considered statistically significant.

Result: (1) There was no significant difference in systolic and diastolic blood pressure, serun glucose (Glu), lipid, renal function, albumin, serum calcium and phosphorus on day of surgery and $1,2,3,4$ weeks after operation $(\mathrm{P}>0.05)$. (2) The concentration of ET-1 and NO were significantly decreased 1 week after operation compared with those on day of surgery. (3) The concentration of ET-1 and NO were significantly increased 2 weeks after operation compared with those of 1 week after operation, but they still below those on day of surgery $(\mathrm{P}<0.05)$. (4) The concentration of ET-1 and NO were significantly increased 3 weeks after operation compared with those of 2 weeks after operation $(P<0.05)$. (5) The concentration of ET-1 and NO were relatively stable 4 weeks after operation compared with those of 3 weeks after operation and they were flat compared with those on day of surgery $(P>0.05)$. (6) The diameters of radial artery trunk and cephalic vein trunk became wider after operation.

Annual Congress of the Chinese Blood

Purification Center Administration

Committee
The blood flow rate became faster after operation and it tend to be flat 3 weeks after operation.

Conclusion: (1) The concentration of ET-1 and NO were declined during the first week after the operation of establishing AVF and then tend to increase 2 weeks after operation. 3 weeks after operation the concentration of ET-1 and NO returned to the level of preoperation. There was no significant changes duing the fourth week after operation. (2) 3 weeks after operation of establishing AVF, vascular endothelial injury and repair was substantially complete, fistula blood flow rose gently and meet the needs of puncture.

Key Words: End-Stage Renal Disease; Atherosclerosis; Matrix Metalloproteinase-10; Tissue Inhibitor of Metalloproteinase-1; carotid Intima Media Thickness.

\section{9 \\ Mortality Risk Analysis in Incident Hemodialysis Patients in Beijing Area \\ Xin-ju Zhao, Liang-ying Gan, Mei Wang, Li Zuo* for the expert team of Beijing Hemodialysis Quality Control and Improvement Center \\ Peking University People's Hospital, Renal Department, 11 Xizhimennan Street, Xicheng District, Beijing, 100044, China \\ Corresponding author: Zuo Li \\ E-Mail: ZuoLi@bjmu.edu.cn}

Objects: Hemodialysis (HD) therapy improves the life expectancy of patients with end stage renal disease (ESRD). However, the adjusted rates of all-cause mortality were 6.5-7.9 times greater for dialysis patients than for individuals in the general population. We studied the mortality rate (including early mortality risk) and pattern in incident HD patients in Beijing area where a relatively complete HD registry is in place.

Methods: We retrospectively review all the incident maintenance HD patients registered in the Beijing Hemodialysis Quality Control and Improvement Center (BJHDQCIC) from January 1, 2007 to December 31, 2012. All included patients were followed to the end of 2013. Patients' demographics, primary cause of end stage renal disease (ESRD), date of first HD, date of death, cause for death, date and cause of censoring were extracted from the registry database. The risk of all-cause mortality was calculated stratified by sex, age, primary cause of ESRD and dialysis vintage.

Results: 11,955 patients were included and 2253 patients censored, 6738 (56.4\%) were males and 5217 (43.6\%) were females. The mean age at dialysis initiation was $57.7 \pm 16.1$ years (range 18.0-99.5 y). The median follow-up time was 19.8 months (range 1-84 months). Diabetes as the cause of ESRD accounted for $29.5 \%$ of patients. There were total 2555 deaths. The overall mortality rate was 8.2 per 100 patient-years. The mortality rates for men and women were 7.9 and 8.5 per 100 patient-years. Mortality rates were 2.9, 5.2, $7.5,10.8$ and 19.2 per 100 patient-years for each age group $(<45 \mathrm{y}$, $45-55,55-64 \mathrm{y}, 65-74 \mathrm{y}$ and $\geq 75 \mathrm{y}$ ), respectively. Generally, the mortality rates were 10.1 in diabetic patients and 7.3 in nondiabetic patients. The mortality rate was lowest in CGN group and highest in other and unknown causes group. The mortality rate in the first year after dialysis initiation was 11.4 per 100 patient-years. We also esti-

Committee 
Fig. 1. The overall mortality rates increased with age (for Abstract 19).
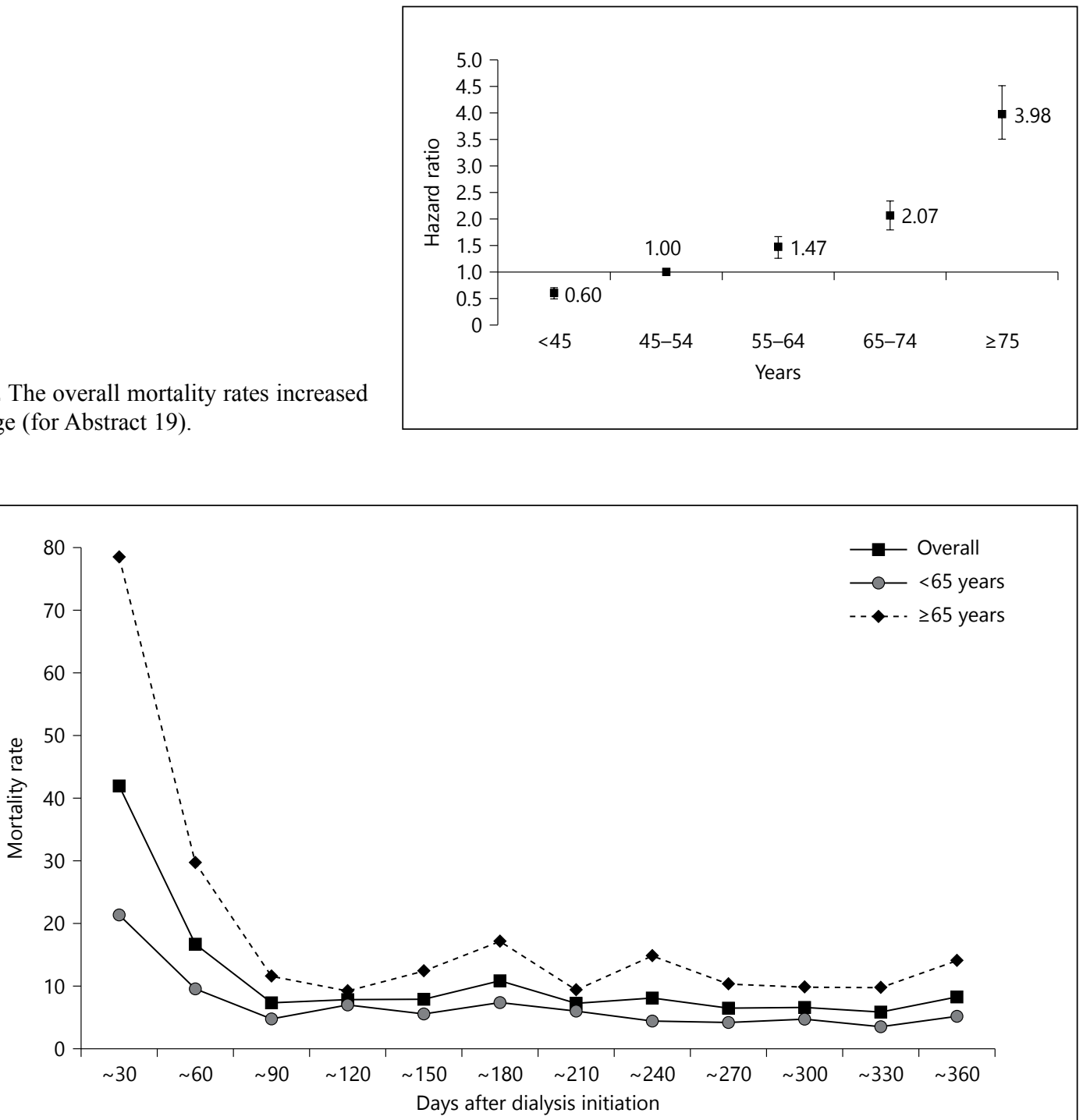

Fig. 2. Crude overall mortality and mortality rate by age group in the first year by month (for Abstract 19).

mated crude mortality rates by month. The mortality rates in the first 3 months were 41.9, 16.6 and 7.2 per 100 patient-years.

Conclusions: HD patients in Beijing area experienced a high mortality risk in the first year with an extremely high mortality in the first 2 months. Patients who were older, female, diabetic and with other or unknown causes of ESRD had higher mortality risk.

Key Words: Hemodialysis; Incidence, Mortality rate, Dialysis Outcomes and Practice Patterns Study (DOPPS).
20

\section{Risk Factors for Early Death in Maintenance Dialysis Patients with Hemorrhagic Stroke}

Ben-gang Huo, Ju-rong Yang, Wei Tan, Jie Yang, Li-rong Lin Lin, Yun-yan Wang, Ming-yu Cai, Long Huang, Ya-ni He*

Department of Nephrology, Daping Hospital, Third Military Medical University, Chongqing, 400042, China

Corresponding author: Ya-ni He

E-Mail: heynmail@yahoo.com

Objective: Hemorrhagic stroke (HS) is a severe complication causing death and disability in maintenance dialysis patients. This stuy retrospectively investigated the incidence rate, 30-day mortal- 
Table 1. Demographics of Beijing incident MHD patients (for Abstract 19)

\begin{tabular}{|c|c|c|c|}
\hline & Beijing cohort & $\begin{array}{l}\text { Death } \\
\text { number }\end{array}$ & $\begin{array}{l}\text { Mortality } \\
\text { rate* }\end{array}$ \\
\hline Number of patients & 11,955 & 2,555 & 8.2 \\
\hline Age (mean, m) & $57.7 \pm 16.1$ & & \\
\hline$<45$ & $2,626(22.0 \%)$ & 201 & 2.9 \\
\hline $45-54$ & $2,371(19.8 \%)$ & 354 & 5.2 \\
\hline $55-64$ & $2,602(21.8 \%)$ & 537 & 7.5 \\
\hline $65-74$ & $2,422(20.3 \%)$ & 698 & 10.8 \\
\hline$\geq 75$ & $1,934(16.2 \%)$ & 765 & 19.2 \\
\hline \multicolumn{4}{|l|}{ Gender } \\
\hline Male & $6,738(56.4 \%)$ & 1,371 & 7.9 \\
\hline Female & $5,217(43.6 \%)$ & 1,184 & 8.5 \\
\hline \multicolumn{4}{|l|}{ Cause of ESRD } \\
\hline Diabetes & $3,531(29.5 \%)$ & 952 & 10.1 \\
\hline Nondiabetic & $8,424(70.5 \%)$ & 1,603 & 7.3 \\
\hline CGN & $3,220(26.9 \%)$ & 420 & 4.3 \\
\hline HT & $2,282(19.1 \%)$ & 485 & 8.3 \\
\hline Unknown & $1,768(14.8 \%)$ & 447 & 12.3 \\
\hline Other causes & $1,154(9.7 \%)$ & 251 & 9.3 \\
\hline \multicolumn{4}{|l|}{ Study phases } \\
\hline$\leq 1$ year & 11,955 & 1,116 & 11.4 \\
\hline$>1$ year & 9,065 & 1,439 & 6.7 \\
\hline 1-2 year & 9,065 & 539 & 6.9 \\
\hline $2-3$ year & 6,614 & 389 & 6.9 \\
\hline 3-4 year & 4,692 & 256 & 6.5 \\
\hline$\geq 5$ year & 3,170 & 255 & 6.2 \\
\hline
\end{tabular}

Mortality rate*: death number per 100 patient-years. MHD = Maintenance hemodialysis; $\mathrm{y}=$ years old.

ity and the risk factors of death in hemodialysis (HD) and peritoneal dialysis (PD) patients in a single center.

Methods: 711 dialysis patients in our center were enrowed, 29 patients with HS were divided into death group and survival grop by the outcome in 30 days. Clinical and laboratory characteristics were compared in two groups.

Results: 26 HS patients were on HD patients and 3 HS patients were on PD. HS occurred with a predominance in HD patients compared with PD patients $(66.5$ vs. $10.5 / 10,000$ patient-years, $\mathrm{p}=$ 0.002 ). The mortality was $73.1 \%$ in HD patients and $67.7 \%$ in PD patients. In death group, systolic blood pressure (SBP) on admission, diastolic blood pressure (DBP) on admission, size of hemorrhage, incidence rate of intraventricular hematoma and iPTH were significantly higher, Glasgow coma score (GCS) were significantly lower $(\mathrm{p}<0.05)$ than survival group. There were significant correlation between 30-day mortality and SBP on admission $(r=0.562$, $\mathrm{p}=0.004)$, DBP on admission $(\mathrm{r}=0.569, \mathrm{p}=0.004)$, intraventricular hematoma $(\mathrm{r}=0.402, \mathrm{p}=0.042)$, size of hemorrhage $(\mathrm{r}=0.612, \mathrm{p}=$ $0.001)$, iPTH $(\mathrm{r}=0.510, \mathrm{p}=0.011)$, GCS $(\mathrm{r}=-0.567, \mathrm{p}=0.001)$. Kaplan-Meier curve showed dialysis modality post HS had no impact on the survival time of the patients (Log-Rank $p=0.545)$.

Conclusion: The 30-day mortality is associated with blood pressure on admission, severity of hemorrhagic stroke and iPTH level in maintenance dialysis patients with hemorrhagic stroke, and continuous renal replacement therapy or peritoneal dialysis post HS has no impact on early outcome.

Key Words: Hemorrhagic stroke; Maintenance dialysis; Outcome; Risk factors.

Annual Congress of the Chinese Blood

Purification Center Administration

Committee
21

\section{Analysis of the Related Factors of Malnutrition in Elderly Maintenance Hemodialysis Patients}

\author{
Long Huang, Yun-yan Wang*, Lei Feng, Jing Mao \\ Blood Purification Centre, Department of Nephrology, \\ Daping Hospital, Third Military Medical University, \\ Chongqing 400042, China \\ Corresponding author: Yun-yan Wang \\ E-Mail:78905052@qq.com
}

Objective: To analyze the related factors and intervention measures in the elderly patients with long-term maintenance hemodialysis patients with malnutrition.

Methods: The data were collected in order to study the influence factors of nutritional status in hemodialysis patients, through the center of 179 cases of long-term maintenance hemodialysis patients, including male 102, female 77, Age 24 86, mean age $69.4 \pm 4.32$ years. To statistics, we collected the December 2014 of clinical index: albumin (ALB) level, age (years), hemodialysis duration (month), serum calcium $(\mathrm{Ca})$, serum phosphorus $(\mathrm{P})$, intact parathyroid hormone (iPTH), hemoglobin, dialysis adequacy, beta 2 microspheres protein, C reflect protein (CRP), sodium bicarbonate, potassium, Qualcomm dialysis, dialysis and filtration mode. And SPSS19.0 statistical software was used for data entry and statistical analysis. The statistical methods included: 1) Descriptive analysis: Frequency, median, confidence interval, composition ratio, average number and standard deviation. 2) Spearman and cross correlation analysis showed that age $(\mathrm{P}=0.001), \mathrm{Ca}(\mathrm{P}=0.001)$, iPTH $(\mathrm{P}=0.01), \mathrm{CRP}$ $(\mathrm{K}), \mathrm{P}(\mathrm{P}=0.05)$ and other factors were less than 0.05 .3$)$ finally the correlation factor is used to the logistic regression analyze.

Results: Through multiple logistic regression analysis, $\mathrm{CRP}(\mathrm{P}=$ $0.022 \mathrm{OR}=1.031$, Logistic, 95\% CI (1.0041058)). The main influencing factors of hypoproteinemia in elderly patient was CRP.

Conclusion: The Logistic regression analysis indicated CRP was the main factor affecting the compliance of patients ALB. Our results indicated $r=-0.2$, it showed that CRP inversely related to ALB (in Table 1), this means that the higher CRP, the lower ALB. CRP is the main indicator of inflammation, and it also shows that the degree of inflammation of the patients can cause malnutrition in patients. how to improve the prevention and treatment of the micro-inflammatory state is the key method to improve the malnutrition in MHD patients.

Key Words: Malnutrition; CRP; Micro-inflammatory state; hypoproteinemia. 